\title{
Strong Phase Measurements - Towards $\gamma$ at CLEO-C
}

\section{Andrew Powell* for the CLEO Collaboration}

University of Oxford, Denys Wilkinson Building, Oxford, OX1 3RH, United Kingdom

E-mail: a.powelllephysics.ox.ac.uk

Strategies that utilise the interference effects within $B \rightarrow D K$ decays hold great potential for improving our sensitivity to the CKM angle $\gamma$. However, in order to exploit fully this potential, knowledge of parameters associated with the $D$ decay, such as strong-phase differences, are required. This essential information can be obtained from the unique quantum-correlated $\psi(3770)$ datasets at CLEO-c. Results of such analyses involving the decay modes $D \rightarrow K \pi, K \pi \pi^{0}, K \pi \pi \pi$ and $K_{S}^{0} \pi \pi$ will be presented.

12th International Conference on B-Physics at Hadron Machines - BEAUTY 2009

September 07 - 112009

Heidelberg, Germany

\footnotetext{
* Speaker.
} 


\section{Introduction}

A theoretically clean method to extract the CKM-angle $\gamma$ is to exploit the interference present in $B^{ \pm} \rightarrow D K^{ \pm}$, where the $D$ is a $D^{0}$ or $\bar{D}^{0}$ decaying to a common final state, $f$. Decay rates in these channels are sensitive to the following amplitude ratios

$$
\frac{A\left(B^{-} \rightarrow \bar{D}^{0} K^{-}\right)}{A\left(B^{-} \rightarrow D^{0} K^{-}\right)}=r_{B} e^{i\left(\delta_{B}-\gamma\right)}, \frac{A\left(B^{+} \rightarrow D^{0} K^{+}\right)}{A\left(B^{+} \rightarrow \bar{D}^{0} K^{+}\right)}=r_{B} e^{i\left(\delta_{B}+\gamma\right)}
$$

which are functions of three parameters: the ratio of the absolute magnitudes of the amplitudes, $r_{B}$; a $C P$-invariant strong-phase difference, $\delta_{B}$; and the weak phase $\gamma$. A variety of $\gamma$ extraction strategies have been suggested depending on the $D$ final state. Final states that can be used are: two-body modes such as $K^{+} K^{-} / \pi^{+} \pi^{-}[1,2], K^{ \pm} \pi^{\mp}$ [3], as well as multi-body final states such as $K_{S}^{0} \pi^{+} \pi^{-}[4,5]$ and $K^{ \pm} \pi^{\mp} \pi^{0} / K^{ \pm} \pi^{\mp} \pi^{+} \pi^{-}[6] .{ }^{1}$ In all cases, the measurement of $\gamma$ is affected by properties of the $D$ decay amplitude. In order to exploit fully the sensitivity to the $B$-specific parameters $\left(r_{B}, \delta_{B}\right.$ and $\left.\gamma\right)$ it is, therefore, highly advantageous to have prior knowledge of the parameters associated with the $D$ decay. This is where CLEO-c plays a crucial role.

These proceedings describe three sets of measurements performed by CLEO-c of $D$-specific parameters relevant to the measurement of $\gamma$. Sec. 2 introduces the $D$ parameters of interest in the context of the $B$ decay rates. Sec. 3 then explains how one can exploit quantum-correlations at the $\psi(3770)$ in order to probe these $D$ parameters. Sec. 4 describes the CLEO-c experiment and data sets used for the analyses. Secs. 5, 6 and 7 describe the experimental procedure and results.

\section{D Parameters Associated with the ADS Method}

In the case of the so-called ADS method [3], where $f=K^{ \pm} \pi^{\mp}, D$-specific parameters contribute to the suppressed $B^{ \pm}$decay-rates as follows:

$$
\Gamma\left(B^{ \pm} \rightarrow\left(K^{\mp} \pi^{ \pm}\right)_{D} K^{ \pm}\right) \propto r_{B}^{2}+\left(r_{D}^{K \pi}\right)^{2}+2 r_{B} r_{D}^{K \pi} \cos \left(\delta_{B}+\delta_{D}^{K \pi} \pm \gamma\right),
$$

where $r_{D}^{K \pi}$ and $\delta_{D}^{K \pi}$ are analogous to the $B^{ \pm}$parameters $r_{B}$ and $\delta_{B} ; r_{D}^{K \pi}$ is the absolute ratio of the doubly Cabibbo suppressed (DCS) to Cabibbo favoured (CF) amplitudes and $\delta_{D}^{K \pi}$ is the corresponding $D$ strong-phase difference. Futhermore, the extended method [6], which considers multi-body ADS modes i.e. $f=\left\{K^{ \pm} \pi^{\mp} \pi^{0}, K^{ \pm} \pi^{\mp} \pi^{+} \pi^{-}\right\}$, introduces an additional $D$ parameter, $R_{f}$, the coherence factor:

$$
\Gamma\left(B^{ \pm} \rightarrow(\bar{f})_{D} K^{-}\right) \propto r_{B}^{2}+\left(r_{D}^{f}\right)^{2}+2 r_{B} r_{D}^{f} R_{f} \cos \left(\delta_{B}+\delta_{D}^{f} \pm \gamma\right)
$$

where $R_{f}$ satisfies the condition $\left\{R_{f} \in \mathbb{R} \mid 0 \leq R_{f} \leq 1\right\}$. This dilution term results from accounting for the resonant sub-structure of the multi-body mode. For modes whose intermediate resonances interfere constructively, $R_{f}$ tends to unity, however if the resonances interfere destructively, then $R_{f}$ tends to zero.

\footnotetext{
${ }^{1}$ For a review of all these methods, and a summary of current and future $B^{ \pm} \rightarrow D K^{ \pm} \gamma$ measurements, see Refs. [7] and [8].
} 


\section{Quantum Correlations at the $\psi(3770)$}

Determination of strong-phase differences and coherence factors can be made from analysis of quantum-correlated $D^{0} \bar{D}^{0}$ pairs. Such an entangled state, with $C=-1$, is produced in $e^{+} e^{-}$ collisions at the $\psi(3770)$ resonance. To conserve this charge-conjugation state, the final state of the $D^{0} \bar{D}^{0}$ pair must obey certain selection rules. For example, both $D^{0}$ and $\bar{D}^{0}$ cannot decay to $C P$ eigenstates with the same eigenvalue. However, decays to $C P$-eigenstates of opposite eigenvalue are enhanced by a factor of two. More generally, final states that are accessible by both $D^{0}$ and $\bar{D}^{0}$ (such as $K^{-} \pi^{+}$) are subject to similar interference effects. Consequently, by considering timeintegrated decay rates of double tag (DT) events, where both the $D^{0}$ and the $\bar{D}^{0}$ are reconstructed, one is sensitive to interference dependent parameters such as strong-phases and coherence factors. Furthermore, these decay rates are also sensitive to charm mixing. Charm mixing is described by two dimensionless parameters: $x \equiv\left(M_{1}-M_{2}\right) / \Gamma$ and $y \equiv\left(\Gamma_{1}-\Gamma_{2}\right) / 2 \Gamma$, where $M_{1,2}$ and $\Gamma_{1,2}$ are the masses and widths, respectively, of the neutral $D$ meson $C P$-eigenstates. The explicit dependence on the mixing parameters can be seen by considering the generalised, time-integrated, DT rate. That is, for a $D^{0} \bar{D}^{0}$ pair decaying to the final state $(f, g)$ :

$$
\Gamma(f \mid g)=Q_{M}\left|A_{f} \bar{A}_{g}-\bar{A}_{f} A_{g}\right|^{2}+R_{M}\left|A_{f} A_{g}-\bar{A}_{f} \bar{A}_{g}\right|^{2},
$$

where $A_{i} \equiv\left\langle i \mid D^{0}\right\rangle, \bar{A}_{i} \equiv\left\langle i \mid \bar{D}^{0}\right\rangle$. The coefficients $Q_{M}$ and $R_{M}$ posses the dependence on the mixing parameters, where $Q_{M} \equiv 1-\left(x^{2}-y^{2}\right) / 2$ and $R_{M} \equiv\left(x^{2}+y^{2}\right) / 2$ [11].

\subsection{Probing strong-phases and coherence factors}

Letting $f$ represent the signal $D$ decay of interest, it is possible to obtain access to strongphases and coherence factors by considering specific states of the 'tag', $g$. As an example, we demonstrate here how sensitivity to strong-phases can be obtained by considering $g$ to be in a $C P$ eigenstate with eigenvalue $\lambda_{C P}$. For the purpose of this discussion, we simplify the problem by ignoring $D$-mixing effects, i.e. $x, y \rightarrow 0$. In this scenario, $Q_{M} \rightarrow 1, R_{M} \rightarrow 0$. Consequently, for $f=K^{-} \pi^{+}$, Eqn.(3.1) reduces to:

$$
\begin{aligned}
\Gamma\left(K^{-} \pi^{+} \mid C P\right) & \propto\left|A_{K \pi} A_{C P}-\bar{A}_{K \pi} A_{C P}\right|^{2} \\
& =\left|A_{K \pi}\right|^{2}\left|A_{C P}\right|^{2}\left(1+\left(r_{D}^{K \pi}\right)^{2}-2 \lambda_{C P} r_{D}^{K \pi} \cos \left(\delta_{D}^{K \pi}\right)\right) .
\end{aligned}
$$

Therefore, with a knowledge of $\left|A_{K \pi}\right|,\left|A_{C P}\right|$ and $r_{D}^{K \pi}$, the observed asymmetry between the rates for $\lambda_{C P}=+1$ and $\lambda_{C P}=-1$ provides direct sensitivity to $\cos \left(\delta_{D}^{K \pi}\right)$. When a multi-body signal mode is considered, such as $f=\left\{K^{ \pm} \pi^{\mp} \pi^{0}, \quad K^{ \pm} \pi^{\mp} \pi^{+} \pi^{-}\right\}$, the amplitude $A_{f}$ must be integrated over all phase-space. This has the effect of modifying Eqn. (3.2) through the transformation $\cos \left(\delta_{D}^{f}\right) \rightarrow$ $R_{f} \cos \left(\delta_{D}^{f}\right)$. Therefore, for $f=K^{-} \pi^{+} \pi^{0}$ :

$$
\Gamma\left(K^{-} \pi^{+} \pi^{0} \mid C P\right)=\left|A_{K \pi \pi^{0}}\right|^{2}\left|A_{C P}\right|^{2}\left(1+\left(r_{D}^{K \pi \pi^{0}}\right)^{2}-2 \lambda_{C P} r_{D}^{K \pi \pi^{0}} R_{K \pi \pi^{0}} \cos \left(\delta_{D}^{K \pi \pi^{0}}\right)\right) .
$$

To give a more concrete overview, expressions from evaluating Eqn. (3.1) are listed in Table 1 for various tag modes against $f=K^{-} \pi^{+}$. As is demonstrated in Ref.[9], while $\left|A_{K \pi}\right|^{2}$ has direct correspondence to the $\mathrm{CF}$ branching fraction $\left(\mathscr{B}_{K \pi}^{C F}\right),\left|\bar{A}_{K \pi}\right|^{2}$ and $\left|A_{C P}\right|^{2}$ possess dependence on the mixing parameters $x$ and $y$, i.e. $\left|\bar{A}_{K \pi}\right|^{2}=\mathscr{B}_{K \pi}^{D C S}(1+\mathscr{O}(x, y))$. Consequently, a linear dependence on $x$ and $y$ is observed in some of the quantum correlated branching fractions quoted in Table 1. 


\begin{tabular}{lc}
\hline \hline Mode & Relative Correlated Branching Fraction \\
\hline$K^{-} \pi^{+}$vs. $K^{-} \pi^{+}$ & $R_{M}$ \\
$K^{-} \pi^{+}$vs. $K^{+} \pi^{-}$ & $\left(1+R_{W}\right)^{2}-4 r \cos \delta_{D}^{K \pi}\left(r \cos \delta_{D}^{K \pi}+y\right)$ \\
$K^{-} \pi^{+}$vs. $C P \pm$ & $1+R_{W S} \pm 2 r \cos \delta_{D}^{K \pi}+y$ \\
$K^{-} \pi^{+}$vs. $e^{-}$ & $1-r y \cos \delta_{D}^{K \pi}-r x \sin \delta_{D}^{K \pi}$ \\
$C P \pm$ vs. $C P \pm$ & 0 \\
$C P+$ vs. $C P-$ & 4 \\
$C P \pm$ vs. $e^{-}$ & $1 \pm y$ \\
\hline \hline
\end{tabular}

Table 1: Correlated $(C=-1)$ effective $D^{0} \bar{D}^{0}$ branching fractions to leading order in $x, y$ and $r^{2}$. The rates are normalised to the multiple of the uncorrelated branching fractions. Some rates show dependence to the wrong-sign rate ratio, $R_{W S}=r^{2}+r y^{\prime}+R_{M}$, where $y^{\prime}=\left(y \cos \delta_{D}^{K \pi}-x \sin \delta_{D}^{K \pi}\right)$.

\section{CLEO-c}

All measurements presented are made with $e^{+} e^{-} \rightarrow \psi(3770)$ data accumulated at the Cornell Electron Storage Ring (CESR). The CLEO-c detector was used to collect these data. Details of the experiment can be found elsewhere [10]. The total integrated luminosity of the data is $818 \mathrm{pb}^{-1}$, however, only $281 \mathrm{pb}^{-1}$ have been used so far for the measurement of $\delta_{D}^{K \pi}$ presented in Sec. 5.

\section{Measurement of the strong-phase difference in $D \rightarrow K^{-} \pi^{+}$}

The first analysis presented is that of the strong-phase difference in $D \rightarrow K^{-} \pi^{+}$. Implementing the method described in Ref. [11], this analysis has performed the first measurements of $y$ and $\cos \left(\delta_{D}^{K \pi}\right)$ in quantum-correlated $\psi(3770)$ data. By comparing the correlated event yields, whose rates are listed in Table 1, with the uncorrelated expectations, we are able to extract $r^{2}, r \cos \left(\delta_{D}^{K \pi}\right)$, $y$ and $x^{2}$. To achieve this, a knowledge of the relevant uncorrelated branching-ratios are needed. This information is gathered by averaging results of single-tagged yields at the $\psi(3770)$ with external measurements using incoherently-produced $D^{0}$ mesons. In addition, to extract $\cos \left(\delta_{D}^{K \pi}\right)$ from $r \cos \left(\delta_{D}^{K \pi}\right)$, knowledge of $r$ is required. This necessary information is obtained by including $R_{W S}$ and $R_{M}$ as external inputs to the least-squares fit. Furthermore, external measurements of the mixing parameters are used as constraints. All correlations amongst the inputs are accounted for.

The analysis has considered a total of seven $C P$-eigenstates reconstructed against the $K^{ \pm} \pi^{\mp}$ signal mode: $K^{+} K^{-}, \pi^{+} \pi^{-}, K_{s}^{0} \pi^{0}, K_{s}^{0} \omega, K_{s}^{0} \pi^{0} \pi^{0}, K_{s}^{0} \eta$ and $K_{L}^{0} \pi^{0}$. In those DTs without a $K_{L}^{0}$, the signal is identified using two kinematic variables: the beam-constrained mass, $M \equiv \sqrt{E_{\text {Beam }}^{2}-\mathbf{p}_{D}^{2}}$, and $\Delta E \equiv E_{D}-E_{\text {Beam }}$, where $E_{\text {Beam }}$ is the beam energy, $\mathbf{p}_{D}$ and $E_{D}$ are the $D^{0}$ candidate momentum and energy, respectively. The reconstruction of $K_{L}^{0} \pi^{0}$ events utilises the missing-mass technique described in Ref. [12]. The analysis finds a result of $\delta_{D}^{K \pi}=\left(22_{-12-11}^{+11+9}\right)^{\circ}$ from using $281 \mathrm{pb}^{-1}$ of data, which is the first direct determination of this phase [13]. An updated result following analysis of the full $818 \mathrm{pb}^{-1}$ dataset is in preparation. 


\section{Measurement of the coherence factor and average strong-phase difference in} $D \rightarrow K^{ \pm} \pi^{\mp} \pi^{0}$ and $D \rightarrow K^{ \pm} \pi^{\mp} \pi^{+} \pi^{-}$

Determination of the average strong-phase difference and associated coherence factors for the modes $f=\left\{K \pi \pi^{0}, K 3 \pi\right\}$ have been made using an analogous technique to that described in Sec. 5 [14]. As shown in Eqn.(3.3), $C P$-tagged multi-body rates provide sensitivity to the product $R_{f} \cos \left(\delta_{D}^{f}\right)$. A means of decoupling these parameters fortunately comes from considering the rate $\Gamma(f \mid f)$. Evaluating Eqn.(3.1) for $g=f$, one obtains:

$$
\Gamma(f \mid f)=Q_{M}\left|A_{f}\right|^{2}\left|\bar{A}_{f}\right|^{2}\left(1-\left(R_{f}\right)^{2}\right)+\left|A_{f}\right|^{4} R_{M}\left(1-2\left(r_{D}^{f}\right)^{2}+\left(r_{D}^{f}\right)^{4}\right) .
$$

In the case of the two-body mode, $f=K^{ \pm} \pi^{\mp}, R_{f}=1$ and Eqn.(6.1) reduces to $\left|A_{f}\right|^{4} R_{M}$ as quoted in Table 1. However, for multi-body final states, one observes that $\left(1-R_{f}^{2}\right)$ is the leading term in Eqn.(6.1). Consequently, the rate $\Gamma(f \mid f)$ provides direct sensitivity to $R_{f}$ and allows for a decoupling of the parameters. All the $C P$-tags listed in Sec. 5 are employed in this analysis, as well as $K_{S}^{0} \phi, K_{S}^{0} \eta^{\prime}$ and $K_{L}^{0} \omega$.

As was done in the $K^{ \pm} \pi^{\mp}$ analysis, a least-squares fit has been used to extract both mixing and strong-phase parameters. Likelihood contours in $R_{f}, \delta_{D}^{f}$ parameter space are shown in Fig. 1(a) for $f=K \pi \pi^{0}$, and Fig. 1(b) for $f=K 3 \pi$. The best-fit values of the coherence factors and average strong-phases are $R_{K \pi \pi^{0}}=0.84 \pm 0.07, \delta_{D}^{K \pi \pi^{0}}=\left(227_{-17}^{+14}\right)^{\circ}, R_{K 3 \pi}=0.33_{-0.23}^{+0.20}$ and $\delta_{D}^{K 3 \pi}=\left(114_{-23}^{+28}\right)^{\circ}$. The uncertainties quoted are a combination of statistical and systematic errors.
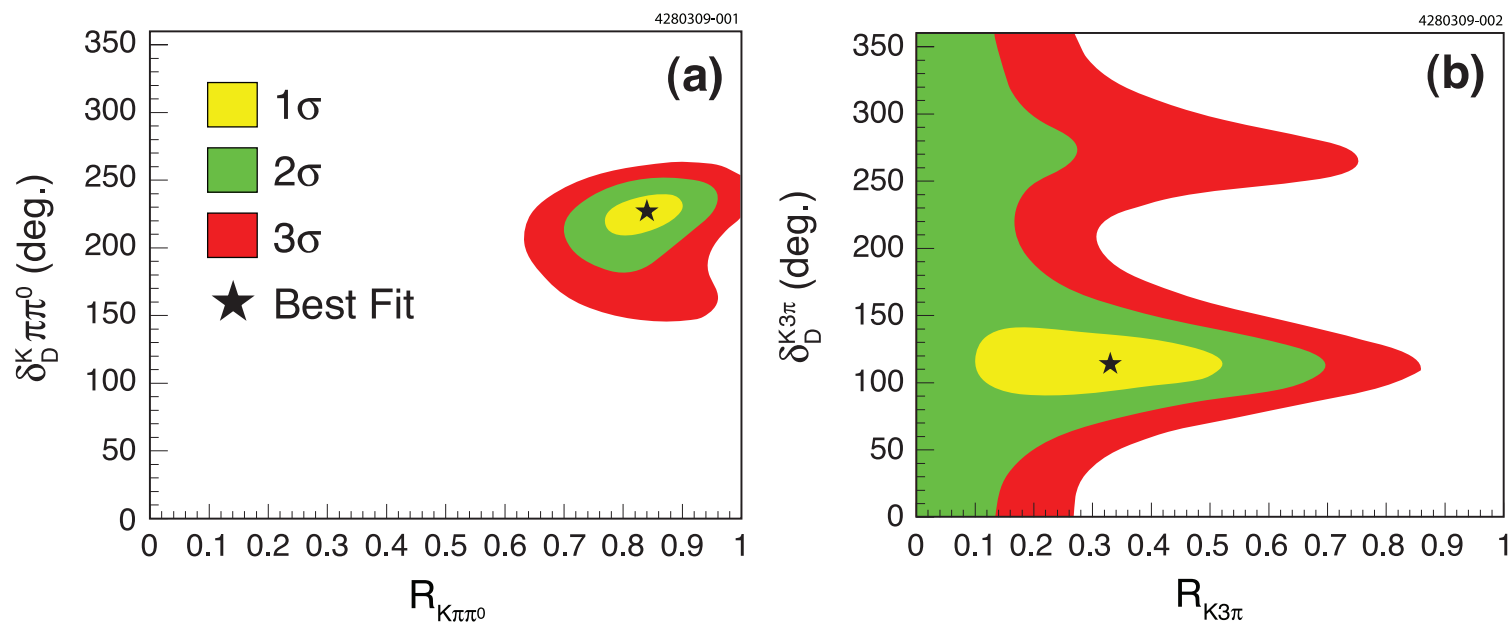

Figure 1: The limits determined on (a) $\left(R_{K \pi \pi^{0}}, \delta_{D}^{K \pi \pi^{0}}\right)$ and (b) $\left(R_{K 3 \pi}, \delta_{D}^{K 3 \pi}\right)$ at the 1,2 and $3 \sigma$ levels.

The results show significant coherence for $D^{0} \rightarrow K \pi \pi^{0}$, but much less so for $D^{0} \rightarrow K \pi \pi \pi$. These results will improve the measurement of $\gamma$ and the amplitude ratio $r_{B}$ in $B^{ \pm} \rightarrow D K^{ \pm}$, where the $D$ decays to $K \pi \pi^{0}$ and $K \pi \pi \pi$. Earlier preliminary results of $R_{K 3 \pi}$ and $\delta_{D}^{K 3 \pi}$ [15] combined with CLEO-c's measurement of $\delta_{D}^{K \pi}$ were shown to improve the expected sensitivity to $\gamma$ at LHCb in a combined ADS analysis of $K \pi$ and $K \pi \pi \pi$ final states by up to $40 \%$ [16].

\section{Measurement of strong-phase variations in $D \rightarrow K_{S}^{0} \pi^{+} \pi^{-}$}

The current best constraints on $\gamma$ come from measurements in $B^{ \pm} \rightarrow D\left(K_{S}^{0} \pi^{+} \pi^{-}\right) K^{ \pm}$and re- 
lated modes [17, 18] by performing likelihood fits to the $K_{S}^{0} \pi^{+} \pi^{-}$Dalitz plot [4]. These fits require models to represent the $D^{0} \rightarrow K_{S}^{0} \pi^{+} \pi^{-}$resonant amplitude structure. Since these models are based on certain assumptions, an inherent systematic uncertainty is associated with this technique. Current estimates predict this error to be between $5^{\circ}$ and $9^{\circ}$, meaning the $\gamma$ measurement would soon become systematically limited at the next generation of flavour-physics experiment. However, an alternative model-independent method has been proposed where events are counted in specified regions of the $K_{S}^{0} \pi^{+} \pi^{-}$Dalitz plot $[4,5]$, thus eliminating the model-uncertainty. This method relies on necessary strong-phase parameters having been determined at CLEO-c.

As Dalitz plot variables we use the invariant-mass squared of the $K_{S}^{0} \pi^{-}$and $K_{S}^{0} \pi^{+}$pairs, which we label as $s_{-}$and $s_{+}$, respectively. The strong-phase at a given point in the $K_{S}^{0} \pi^{+} \pi^{-}$Dalitz plot is then $\delta_{D}\left(s_{-}, s_{+}\right)$. For the phase difference between $D^{0} \rightarrow K_{S}^{0} \pi^{+} \pi^{-}$and $\bar{D}^{0} \rightarrow K_{S}^{0} \pi^{+} \pi^{-}$at the same point in the Dalitz plot, we define

$$
\Delta \delta_{D} \equiv \delta_{D}\left(s_{-}, s_{+}\right)-\delta_{D}\left(s_{+}, s_{-}\right) .
$$

The quantities measured by CLEO-c that provide input to the model-independent $\gamma$ determination are the averages of $\cos \left(\Delta \delta_{D}\right)$ and $\sin \left(\Delta \delta_{D}\right)$ in the $i$ th Dalitz plot bin. We denote these terms $c_{i}$ and $s_{i}$, respectively. In a completely analogous manner to the analyses presented in Secs. 5 and $6, c_{i}$ can be determined from $C P$-tagged decay rates, while $s_{i}$ is extracted from considering the double Dalitz plot of $K_{S}^{0} \pi^{+} \pi^{-}$vs. $K_{S}^{0} \pi^{+} \pi^{-}$. Furthermore, additional constraints on $c_{i}$ and $s_{i}$ are obtained through $K_{L}^{0} \pi^{+} \pi^{-}$events.

The choice of Dalitz plot binning affects the statistical precision of the analysis. It has been demonstrated in Ref. [5] that it is beneficial to choose bins such that $\Delta \delta_{D}$ varies as little as possible across each bin. The binning used in this analysis, with eight-pairs of bins uniformly dividing $\Delta \delta_{D}$ over the range $[0,2 \pi]$, is shown in Fig. 2(a). The location of these bins in phase space are chosen based on the BaBar isobar model given in Ref. [19].

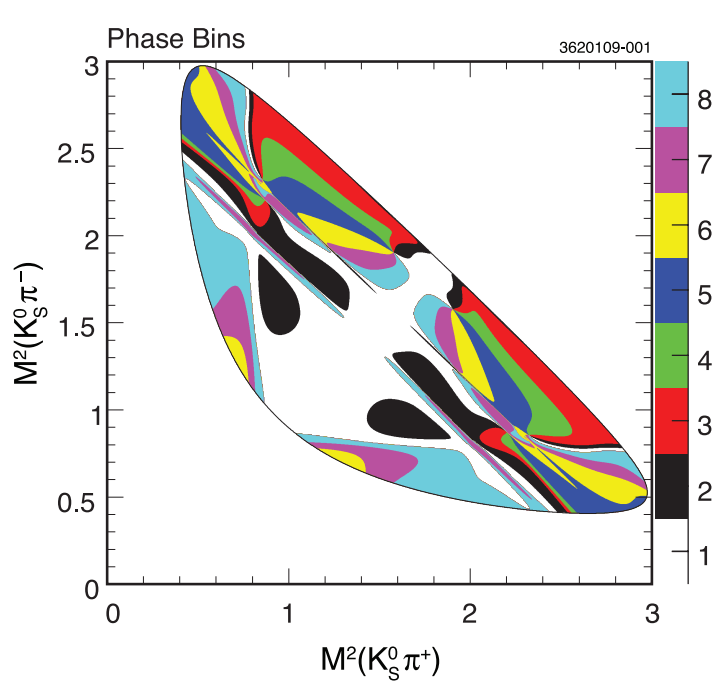

(a)

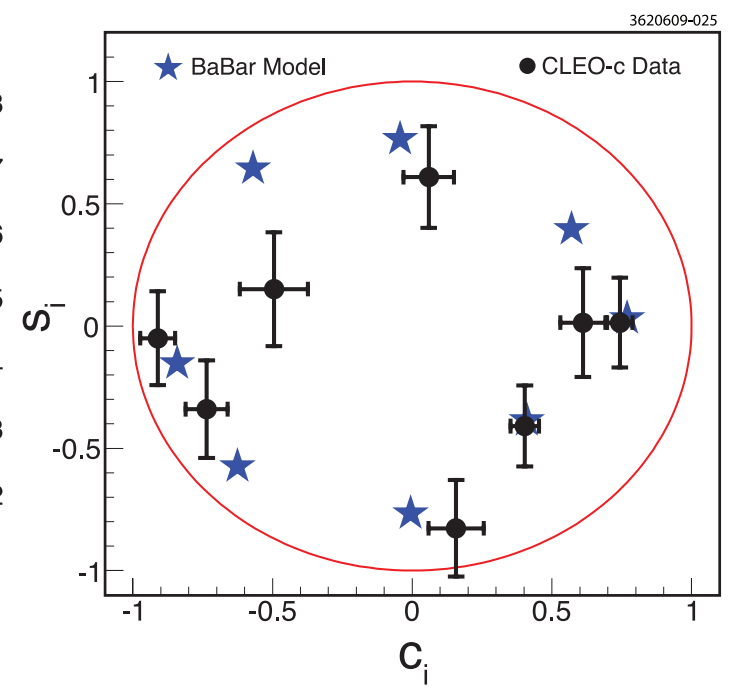

(b)

Figure 2: In (a), the uniform $\left|\Delta \delta_{D}\right|$ binning of the $K_{S}^{0} \pi^{+} \pi^{-}$Dalitz plot. In (b), the comparison of the measured $c_{i}$ and $s_{i}$ (circles with error bars) to the predictions from the BaBar isobar model (stars). 
The values of $c_{i}$ and $s_{i}$ from the combined analysis of $K_{S}^{0} \pi^{+} \pi^{-}$and $K_{L}^{0} \pi^{+} \pi^{-}$tagged events are shown graphically in Fig 2(b). When used as input to the $\gamma$ measurement, these results are expected to replace the current model uncertainty of $5^{\circ}-9^{\circ}$ with an uncertainty due to the statistically dominated error on $c_{i}$ and $s_{i}$ of $1.7^{\circ}[20]$.

\section{Conclusion}

The importance of CLEO-c's quantum-correlated $\psi(3770)$ dataset in the context of measuring the CKM angle $\gamma$ has been described. Analysis of a variety of two- and multi-body $D^{0}$ decays with these data have provided vital measurements of $D^{0}$ strong-phases, and associated parameters, for model-independent $\gamma$ measurements at LHCb. In addition to the modes presented here, results are in preparation for other promising final states, such as $D^{0} \rightarrow K_{S}^{0} K^{+} K^{-}$.

\section{References}

[1] M. Gronau and D. London, Phys. Lett. B 253 (1991) 483.

[2] M. Gronau and D. Wyler, Phys. Lett. B 265 (1991) 172.

[3] D. Atwood, I. Dunietz and A. Soni, Phys. Rev. Lett. 78 (1997) 3257.

[4] A. Giri, Y. Grossman, A. Soffer and J. Zupan, Phys. Rev. D 68 (2003) 054018.

[5] A. Bondar and A. Poluektov, Eur. Phys. J. 47 (2006) 347.

[6] D. Atwood and A. Soni, Phys. Rev. D 68 (2003) 033003.

[7] K. Miyabayashi, these proceedings.

[8] S. Ricciardi, these proceedings.

[9] Z.Z. Xing, Phys. Rev. D 55 (1997) 196.

[10] Y. Kubota et al., Nucl. Instrum. Meth. Phys. Res., Sect. A 320, (1992) 66; D. Peterson et al., Nucl. Instrum. Meth. Phys. Res., Sect. A 478, (2002) 142.

[11] D. Asner and W. Sun, Phys. Rev. D 73, (2006) 034024; 77 (2008) 019901(E).

[12] Q. He et al.[CLEO Collaboration], Phys. Rev. Lett. 100 (2008) 091801.

[13] J. Rosner et al. [CLEO Collaboration], Phys. Rev. Lett. 100 (2008) 221801, D. Asner et al. [CLEO Collaboration] Phys. Rev.D 78 (2008) 012001.

[14] N. Lowery et al. [CLEO Collaboration], Phys. Rev. D 80 (2009) 031105(R).

[15] A. Powell [CLEO Collaboration], in Fundamental Interactions: Proceedings of the 23rd Lake Louise Winter Institute 2008, World Scientific Publishing.

[16] K. Akiba at al. CERN Report No. CERN-LHCb-2008-031 (2008).

[17] K .Abe et al. [Belle Collaboration], arXiv:0803.3375[hep-ex]

[18] B. Aubert et al. [BaBar Collaboration], Phys. Rev. D 78 (2008) 034023.

[19] B. Aubert et al. [BaBar Collaboration], arXiv:hep-ex/0607104.

[20] R. Briere et al. [CLEO Collaboration], Phys. Rev. D 80 (2009) 032002. 